\section{First-trimester exposure to ACE inhibitors is associated with congenital malformations}

Women should not be given angiotensinconverting-enzyme (ACE) inhibitors during the second and third trimesters of pregnancy, as these drugs have been linked with an increased risk of fetopathy. Data on the use of ACE inhibitors in the first trimester are sparse, however, and researchers in the US have addressed this issue using data from 29,507 births recorded in the Tennessee Medicaid database.

In total, 411 infants had been exposed to antihypertensive medications during the first trimester alone; 209 of these had been exposed to ACE inhibitors and 202 to other hypertensive medications. Overall, 856 infants were diagnosed with at least one major congenital malformation not related to a chromosomal defect or a clinical genetic syndrome. The most common types of malformation were cardiovascular, musculoskeletal and gastrointestinal malformations, affecting 305, 195 and 119 infants, respectively. The risk of major congenital malformation was over twofold higher in infants who had been exposed to ACE inhibitors in the first trimester alone than in those not exposed to antihypertensive medications (risk ratio 2.71). This increased risk results mainly from higher risks of cardiovascular and central nervous system malformations (risk ratios 3.72 and 4.39, respectively); other malformations were not significantly higher in infants exposed to ACE inhibitors. Exposure to antihypertensive medications other than ACE inhibitors in the first trimester had no effect on the risk of any major congenital malformation.

The authors conclude that ACE inhibitors are associated with an increased risk of a major congenital malformation and should not be used during pregnancy.

Original article Cooper WO et al. (2006) Major congenital malformations after first-trimester exposure to ACE inhibitors. N Engl J Med 354: 2443-2451

\section{HIV-infected patients are suitable candidates for renal transplantation}

Since the introduction of highly active antiretroviral therapy (HAART) in 1996, survival of people infected with HIV has markedly improved. With increasing longevity has come an increase in the incidence of chronic disease, including end-stage renal disease. Given that use of immunosuppressants in HIV-positive patients can increase morbidity and mortality, renal transplantation in this population has been considered to be an inefficient use of scarce organs. New data indicate that reluctance to transplant HIV-positive patients is no longer warranted.

Qiu and colleagues analyzed kidney transplant outcomes data from the United Network for Organ Sharing (1997-2004). Graft and patient survival for HIV-positive recipients $(n=38)$ were compared with equivalent data from HIV-negative transplantees $(n=38)$ who had received a kidney from the same donor.

The analysis detected no significant difference in any outcome between groups. In fact, there was a trend towards better graft and patient survival at 5 years among HIV-positive patients compared with their non-infected counterparts (graft survival $76.1 \%$ vs $65.1 \%$, respectively, $P=0.21$; patient survival $91.3 \%$ vs $87.3 \%$, respectively, $P=0.72$ ). Serum creatinine levels were slightly, but not significantly, higher in HIV-positive graft recipients 1, 3 and 5 years after transplantation. Incidences of delayed graft function and acute rejection were similar in the two groups. In HIV-infected patients, more grafts failed because of patient death than as a result of rejection.

The results of this study indicate that transplanting HIV-positive patients with end-stage renal disease in the HAART era is ethical and efficacious.

Original article Qiu J et al. (2006) HIV-positive renal recipients can achieve survival rates similar to those of HIV-negative patients. Transplantation 81: 1658-1661

\section{Long-term steroid therapy preserves renal function in sarcoid tubulointerstitial nephritis}

Corticosteroids are the mainstay of therapy for granulomatous tubulointerstitial nephritis, an uncommon complication of sarcoidosis. Treatment improves renal function in the short term, but there is a dearth of data on the longterm outcome of maintenance therapy in patients with this condition. 